\title{
Exotic phases in geometrically frustrated triangular Ising magnets
}

\author{
Ying Jiang ${ }^{1}$ and Thorsten Emig ${ }^{2}$ \\ ${ }^{1}$ Département de Physique, Université de Fribourg, Chemin du Museé 3, CH-1700 Fribourg, \\ Switzerland \\ ${ }^{2}$ Laboratoire de Physique Théoretique et Modèles Statistiques, CNRS UMR 8626 , \\ Université Paris-Sud, 91405 Orsay, France \\ E-mail: ying.jiang@unifr.ch
}

\begin{abstract}
We report a systematic study of both quantum and classical geometrically frustrated Ising models with competing ordering mechanism. The ordering comes in the classical case from a coupling of two-dimensional (2D) layers and in the quantum model from the quantum dynamics induced by a transverse field. We develop a microscopic derivation of the Landau-Ginzburg-Wilson (LGW) Hamiltonian for these models and show that it can be interpreted as the free energy of three-dimensional (3D) elastic non-crossing strings. By utilizing this effective Hamiltonian, the entire transverse field versus temperature phase diagram for the 2D quantum Ising model is obtained analytically, including the universality classes of both the quantum and the finite temperature transitions. The structures of the ordered phases in both 3D classical and 2D quantum Ising models are obtained from a detailed entropy argument. The results are in excellent agreement with recent numerical simulations.
\end{abstract}

The competition of quantum and thermal fluctuations is crucial in finding and understanding exotic phases in geometrically frustrated magnets $[1,2]$. For geometrically frustrated Ising system, the macroscopic degeneracy of the classical ground state may endow the system with a continuous symmetry which precludes an ordered phase at finite temperatures for 2D quantum magnets due to the Mermin-Wagner theorem [3]. Another possible but contrary scenario is 'order-from-disorder' [4] where quantum fluctuations select a small particularly susceptible class of the ground-state manifold and yield an ordered symmetry-broken state [5]. Hence one expects that weak competing fluctuations about the classical ground states is able to generate new strongly correlated states and phase transitions of unexpected universality classes.

The antiferromagnetic Ising model on a triangular lattice (TIAF)

$$
H=J \sum_{\langle i, j\rangle} \sigma_{i} \sigma_{j}
$$




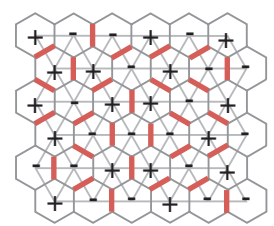

(a)

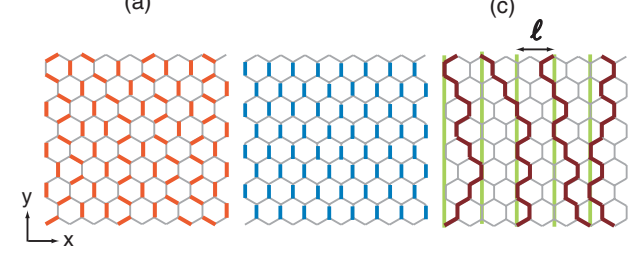

(b)

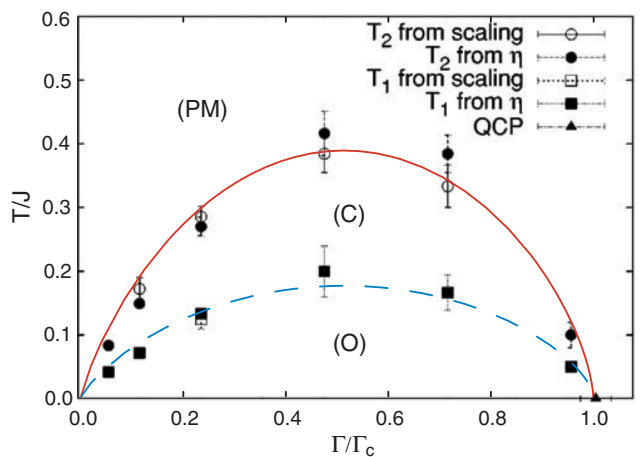

(d)

Figure 1. (a) Relation between spins and dimers. (b) Mapping of dimers to strings where the numbers denote the height profiles. (c) The two flat states with flippable plaquettes (grey). (d) Phase diagram predicted by equation (7) and Monte Carlo results of figure 1 in [29]. A critical phase (C) is separated by a boundary at $T_{\mathrm{c}, 2}$ from the paramagnetic phase $(\mathrm{PM})$ and at $T_{\mathrm{c}, 1}=4 / 9 T_{\mathrm{c}, 2}$ from an ordered phase $(\mathrm{O})$.

(This figure is in colour only in the electronic version)

the possibly simplest yet nontrivial realization of geometrical frustration, is disordered even at zero temperature with a finite entropy density and algebraic decaying spin correlations [6]. We are interested in two models, 2D quantum TIAF and 3D stacked TIAF; both models are based on the triangular Ising antiferromagnet. Actually, these two models share some common features: by the Suzuki-Trotter theorem [7], the 2D quantum TIAF can be mapped to 3D classical stacked TIAF.

We start first from considering the classical 2D TIAF in the ground states. Since each triangle has exactly one frustrated bond, a complete hard-core dimer covering of the dual hexagonal lattice can be obtained [8]; see figure 1(a). The string representation follows from a given dimer state by the subtraction of a fixed reference state with all vertical bonds occupied [9]; see figure 1(b). The strings fluctuate but remain directed (along the reference direction) and non-crossing (due to the frustration of the spin system); the mean string distance is $\ell=3 a / 2$, with $a$ the triangular lattice constant.

The effective free energy of long-wavelength fluctuations of the string lattice is in the form of a continuum elastic energy

$$
\mathcal{F}_{\text {el }}=\int \mathrm{d}^{2} \mathbf{r}\left\{\frac{c_{11}}{2}\left(\partial_{x} u\right)^{2}+\frac{c_{44}}{2}\left(\partial_{y} u\right)^{2}+V_{L}(u)\right\}
$$

with a periodic potential $V_{L}(u)$ which reflects the discreteness of the lattice and takes the form of $V_{L}=-v \cos \left(4 \pi\left(u-u_{0}\right) / a\right)$ with $v>0 . u$ is the string displacement from a lattice of straight strings. $u_{0}$ is a global offset which differentiates between two non-equivalent classes of straight states that are not related by shifts by a lattice vector of the triangular lattice; see figure 1(c). For the straight line lattice which corresponds to the classical spin configuration $(++-)$ on the three sublattices, the mean string positions are centred between the spins, and the global offset $u_{0}=n a / 2, n \in \mathbb{Z}$. For the flat string state in which the mean string positions are located on the sites of one sublattice which has the same number of + and - spins, i.e., zero magnetization as indicated by 0 in figure $1(\mathrm{c}), u_{0}=a / 4+n a / 2$. The compression $\left(c_{11}\right)$ and tilt $\left(c_{44}\right)$ moduli of the corresponding elastic system are both of entropic 
origin. For $\sqrt{c_{11} c_{44}}>2 \pi / a^{2}$ the potential $V_{L}$ is relevant, and the strings lock into that class of states with the maximal number of configurations which yields at arbitrary large $\mathbf{r}$ still a finite displacement $\left\langle[u(\mathbf{r})-u(\mathbf{0})]^{2}\right\rangle_{\text {flat }} \sim$ const, where the displacement is measured relative to the respective flat state. This entropy argument shows [10] that the states of orientation $(+-0)$ allow for a larger number of localized configurations, and hence is selected and $u_{0}=-a / 4$ in the locking potential. The compression modulus can be obtained from the equivalence of non-crossing strings to 1D free Fermions [11] which is based on the Pauli principle and yields $c_{11}=\pi^{2} \rho^{3} / g$ with $g=c_{44} / \rho$ the string tension. Then the periodic potential $V_{L}$ is irrelevant since $\sqrt{c_{11} c_{44}}=\pi \rho^{2}<2 \pi / a^{2}$. However, in other models additional interactions may increase the string stiffness, and the lock-in potential might become relevant.

Within the string picture, the classical spin-spin correlations can easily be obtained just by counting the number of strings in between [12], i.e., $\left\langle\sigma_{i} \sigma_{j}\right\rangle=\left\langle(-1)^{\#_{\text {line }}\left[x_{j}, x_{i}\right]}\right\rangle$. This transforms the problem of spin-spin correlation to a corresponding problem of string displacement field correlation, yielding the known exact result of $\left\langle\sigma_{i} \sigma_{j}\right\rangle=\left(r_{i j} / a\right)^{-\eta} \cos \left(2 \pi r_{i j} / 3 a\right), \eta=1 / 2$.

As in the 2D system, for the stacked TIAF whose Hamiltonian reads $H_{3 \mathrm{D}}=$ $J_{\|} \sum_{\langle i j\rangle, k} \sigma_{i k} \sigma_{j k}-J_{\perp} \sum_{i, k} \sigma_{i k} \sigma_{i k+1}\left(J_{\|}, J_{\perp}>0\right.$ and $\langle i j\rangle$ indicates summation over nearestneighbour pairs in each TIAF plane, $k$ numbers the layers), the in-plane frustration is expected to have strong influence on the underlying physics due to the Ising symmetry. Experimentally, the stacked model is a reasonable description of triangular cobalt antiferromagnets of the type $\mathrm{ACoX}_{3}$ where $\mathrm{A}$ is an alkali metal and $\mathrm{X}$ a halogen atom [13]. Due to the non-frustration in the additional stacked direction, the increased dimensionality of the stacked system leads to zero entropy per spin at $T=0$ and ordering at even finite temperature might be possible.

The stacked TIAF has been studied first by Blankschtein et al [14, 15]. An LGW Hamiltonian has been guessed by symmetry arguments, leading to a 3D XY model with a six-fold symmetry breaking term; this predicted a XY-like transition into the paramagnetic phase. The sign of the six-fold clock term was not fixed in this approach, and hence two ordered phases with a relevant symmetry breaking term were possible in principle. However, more recent simulations and hard-spin mean field theory indicated the existence of only one ordered phase which corresponds to the one found in [14] at higher temperatures only [16-21]. Moreover, the above LGW approach has been put somewhat into question by Coppersmith [22], mainly since it has been argued that it fails to describe the system at low temperatures by neglecting the restriction to classical spin value \pm 1 and thus geometrical frustration.

In fact, the spin-string mapping mentioned above can also be utilized to the corresponding stacked system: each frustrated spin layer can be mapped to a $2 \mathrm{D}$ elastic string lattice. When considering the nature of the in-plane geometrical frustration, the intra-layer and interlayer spin-spin couplings can be written in terms of string displacement field and lead immediately to a reduced 3D string Hamiltonian $[10,12]$

$$
\begin{aligned}
H_{S}=-\tilde{K}_{\|} \sum_{\langle i j\rangle, k} \cos \left[\frac{\pi}{\ell}\left(u_{i k}-u_{j k}+\eta_{i j} a / 2\right)\right] \\
\quad-\tilde{K}_{\perp} \sum_{i, k} \cos \left[\frac{\pi}{\ell}\left(u_{i k}-u_{i k+1}\right)\right]+v \sum_{i, k} \cos \left[\frac{6 \pi}{\ell} u_{i k}\right]
\end{aligned}
$$

with couplings $\tilde{K}_{\|}=J_{\|} / T, \tilde{K}_{\perp}=J_{\perp} / T$ and mean string separation $\ell=3 a / 2$. The shift $\eta_{i j}$ $(=+1$ for the bond directions $(a, 0)$ and $=-1$ for the directions $(a / 2, \pm \sqrt{3} a / 2))$ reflects the in-plane frustration.

Thus the stacked Ising model maps to a stack of planar lattices of non-crossing strings which is described by a $(2+1) D$ frustrated XY model with a six-fold clock term. However, our effective model differs from the previous LGW theory [14] in two important points: (i) the 
in-plane XY coupling is frustrated and (ii) there is a topological constraint on $\phi$ since $u$ is restricted by the non-crossing condition. The latter point will lead to an entropically increased phase stiffness on large length scales.

The transition of the system to the paramagnetic phase is driven by topological defects which are generated by thermal fluctuations. These defects are superpositions of two type of defect: one type is from fully frustrated triangles with all spins aligned, and another type arises from the mismatch of strings in adjacent layers. Since the stacking [23] and the six-fold clock term [24] are known to be irrelevant at a critical point under renormalization, the transition to a paramagnetic phase must be in the $3 \mathrm{D} X Y$ universality class. In the ordered phase, the $\mathrm{XY}$ couplings of equation (3) can be expanded in $u_{i k}$, and in the continuum limit each layer is described by equation (2) with an additional harmonic interlayer coupling. Moreover, the sixfold clock is relevant, and the strings are locked into a flat state with global offset $a / 4$, leading to a long range order spin state with sublattice magnetization $\langle\sigma\rangle=\left(\frac{\sqrt{3}}{2},-\frac{\sqrt{3}}{2}, 0\right)$. This result appears to be consistent with more recent simulations. In terms of the spin parameter, the ordered phase can be illustrated as following: the spins on two sublattices form ordered stacked honeycomb antiferromagnets where two sublattices have a finite but opposite magnetization; the spins on the third sublattices form Ising chains along the stacking directions. These chains are decoupled from each other and behave as individual chains which drive the magnetization of the third sublattice to zero. Such decoupled chain structure contributes significantly to the specific heat of the system at low temperature. These excitations have actually be seen in Monte Carlo simulations [17].

Now let us turn to the 2D transverse field triangular Ising model

$$
H=J \sum_{\langle i, j\rangle} \sigma_{i}^{z} \sigma_{j}^{z}+\Gamma \sum_{i} \sigma_{i}^{x} .
$$

The Suzuki-Trotter theorem [7] shows that the quantum TIAF can be mapped to $n$ layers classical stacked TIAF with corresponding coupling constants $\tilde{K}_{\|}=J /(n T)$ and $\tilde{K}_{\perp}=$ $\frac{1}{2} \ln (n T / \Gamma)$. The correspondence becomes exact for an infinite Trotter number $n \rightarrow \infty$. Actually, after the mapping, the effective classical 3D Hamiltonian takes exactly the same form as that of the 3D classical stacked Ising model. At $T=0$, the system shows 3D behaviour; it is then straightforward to conclude that the quantum phase transition of the 2D quantum frustrated Ising model is in the $3 \mathrm{D} X Y$ universality class. At $T \neq 0$, the system behaves more like a $2 \mathrm{D}$ one due to the finite size property of the corresponding effective classical system. The transitions can be investigated by rewriting the partition function of equation (3) into a spin wave and a vortex part via the Villain mapping [25, 26]. The Villain couplings $K$ are known in two limits, $K=\tilde{K}$ for $\tilde{K} \rightarrow \infty$ and $K=1 /(2 \ln (2 / \tilde{K}))$ for $\tilde{K} \rightarrow 0$. In fact, these limits are realized for $\tilde{K}_{\perp}$ and $\tilde{K}_{\|}$at large Trotter numbers $n$, leading to $K_{\perp}=\frac{1}{2} \ln (n T / \Gamma), \quad K_{\|}=\frac{1}{2} \ln ^{-1}(2 n T / J)$. At large $n$, we can expand this relation, giving

$$
\sqrt{K_{\|} K_{\perp}}=\frac{1}{2}\left[1-\ln (2 \Gamma / J) K_{\|}\right] .
$$

For layered XY models, dimensional crossover scaling [27] can be used to obtain a relation between the $3 \mathrm{D}$ critical value $K_{\infty}^{\mathrm{c}}$ for the in-plane coupling and the corresponding $2 \mathrm{D}$ critical value $K_{1}^{\mathrm{c}}$,

$$
K_{\infty}^{\mathrm{c}}=K_{1}^{\mathrm{c}}\left(v+\gamma^{-1} \sqrt{K_{\perp} / K_{\|}}\right)^{-1} .
$$

At the quantum critical point the classical string system is a $3 \mathrm{D}$ one, and thus at $K_{\|}=K_{\infty}^{\mathrm{c}}$ the relations of equations (5) and (6) must be identical for consistency. This implies $\gamma=1 /\left(2 K_{1}^{\mathrm{c}}\right)$ and $\Gamma_{\mathrm{c}} / J=\frac{1}{2} \mathrm{e}^{\mathrm{v} / K_{1}^{\mathrm{c}}}$. The usual Kosterlitz-Thouless $(\mathrm{KT})$ argument yields for the vortex unbinding transition $K_{1}^{\mathrm{c}}=2 / \pi \times 2 / \sqrt{3}$ on the triangular lattice [28], leading to $\Gamma_{\mathrm{c}} / J=1.24$. 
However, this estimate neglects renormalization effects due to the clock term, the frustration, and the non-crossing of strings which should provide a net increase of $\Gamma_{\mathrm{c}}$. Interestingly, recent Monte Carlo studies suggest $\Gamma_{\mathrm{c}} / J \approx 1.65$ [29].

At finite temperature, 2D XY physics dominates at large scales, and KT singularities are expected [25]. By making use of finite size scaling, from $K_{\infty}^{\mathrm{c}}$ we obtain the critical coupling $K_{n}^{\mathrm{c}}\left(K_{\perp}\right)$ as a function of $K_{\perp}$ and $n$. This result can be used to determine the vortex unbinding transition at finite $T$. Be aware that, due to equation (5), the renormalization of the coupling constants $K_{\perp}$ and $K_{\|}$must be dependent; this yields in the limit $n \rightarrow \infty$ the phase boundary of a critical phase $(\mathrm{C})$ with bound defects,

$$
\frac{T_{\mathrm{c}, 2}}{J}=b \frac{\Gamma}{\Gamma_{\mathrm{c}}} \ln ^{v}\left(\frac{\Gamma_{\mathrm{c}}}{\Gamma}\right)
$$

where $b$ is a constant determined by the quantum critical value of $\Gamma_{\mathrm{c}}$. At a lower $T_{\mathrm{c}, 1}=$ (4/9) $T_{\mathrm{c}, 2}$ there is a second transition to an ordered state where the clock term becomes relevant and locks the strings to the lattice. Close to the quantum critical point both temperatures vanish $\sim\left(\Gamma_{\mathrm{c}}-\Gamma\right)^{v}$ as expected from scaling. Figure 1 compares equation (7) to recent Monte Carlo data, showing very good agreement across the entire range of $\Gamma$ for $b=0.98$. The spin correlations in phase $\mathrm{C}$ decay with $\eta$ varying continuously between $\eta=1 / 4$ at $T_{\mathrm{c}, 2}$ and $\eta=1 / 9$ at $T_{\mathrm{c}, 1}$ [25]. At the quantum critical point one has the 3D XY result $\eta \approx 0.040$ [30]. The ordered phase $(\mathrm{O})$ is characterized by finite sublattice magnetizations $(\sqrt{3} / 2,-\sqrt{3} / 2,0)$, which is consistent with simulations [29].

Interestingly, the mapping to strings should also be useful in the presence of quenched disorder when the system usually exhibits spin glass behaviour. The behaviour of a 2D string lattice with pinning sites [31] suggests that in geometrically frustrated systems the spin glass correlation function [32] $g_{\mathrm{SG}}\left(\mathbf{r}_{i}-\mathbf{r}_{j}\right)=\overline{\left\langle\sigma_{i} \sigma_{j}\right\rangle^{2}}$ decays at least according to a power law, which implies that the spin glass phase is destroyed by the huge degeneracy from the geometric frustration. However, quenched disorder will induce topological defects, and a full analysis is more complicated. The situation is under better control for geometrically frustrated Ising systems with random dilution. The dilution sites act as pinning centres for the strings and topological defects do not arise from this type of disorder. Another potential application of the spin-string mapping is the study of hard-core bosons on a triangular lattice with nearest neighbour repulsive interaction and hopping. This model can exactly be mapped to XXZ Heisenberg model. Without hopping term, it is simply the classical TIAF. The hopping term (or XY term in spin language) acts as a quantum fluctuation which may induce a similar order from disorder mechanism; hence a phase with simultaneous Ising long-range order and XY order is expected. This ordered phase in spin systems corresponds to an ordered phase in the boson system with simultaneous translational long-range order and off-diagonal long-range order, i.e. the supersolid phase. Evidence of this supersolid phase has been observed in recent numerical simulations [33].

\section{Acknowledgment}

YJ is indebted to Professor Dionys Baeriswyl for fruitful discussion.

\section{References}

[1] Sachdev S 1999 Quantum Phase Transitions (Cambridge: Cambridge University Press)

[2] Diep H T (ed) 1994 Magnetic Systems with Competing Interactions (Singapore: World Scientific)

[3] Mermin N D and Wagner H 1966 Phys. Rev. Lett. 171133 
[4] Villain J et al 1980 J. Physique 411263

[5] Moessner R, Sondhi S L and Chandra P 2000 Phys. Rev. Lett. 844457

Moessner R and Sondhi S L 2001 Phys. Rev. B 63224401

[6] Wannier G H 1950 Phys. Rev. 79357

Houtappel R M F 1950 Physica (Amsterdam) 16425

Stephenson J 1970 J. Math. Phys. 11413

[7] Suzuki M 1976 Prog. Theor. Phys. 561454

[8] Nienhuis B, Hilhorst H J and Blöte H W J 1984 J. Phys. A: Math. Gen. 173559

[9] Zeng C, Middleton A A and Shapir Y 1996 Phys. Rev. Lett. 773204

[10] Jiang Y and Emig T 2006 Phys. Rev. B 73104452

[11] Pokrovsky V L and Talapov A L 1979 Phys. Rev. Lett. 4265

[12] Jiang Y and Emig T 2005 Phys. Rev. Lett. 94110604

[13] Collins M F and Petrenko O A 1997 Can. J. Phys. 75605

[14] Blankschtein D et al 1984 Phys. Rev. B 29 R5250

[15] Netz R and Berker A 1991 Phys. Rev. Lett. 66377

[16] Matsubara F and Inawashiro S 1987 J. Phys. Soc. Japan 562666

[17] Kim J, Yamada Y and Nagai O 1990 Phys. Rev. B 414760

[18] Nagai O, Kang M, Yamada Y and Miyashita S 1994 Phys. Lett. A 196101

[19] Plumer M L and Mailhot A 1995 Physica A 222437

[20] Moessner R, Sondhi S L and Chandra P 2001 Phys. Rev. B 64144416

[21] Akgüç G B and Yalabık M C 1995 Phys. Rev. E 512636

[22] Coppersmith S N 1985 Phys. Rev. B 321584

[23] Korshunov S E 1990 Europhys. Lett. 11757

[24] Aharony A et al 1986 Phys. Rev. Lett. 571012

[25] José J V, Kadanoff L P, Kirkpatrick S and Nelson D R 1977 Phys. Rev. B 161217

[26] Kleinert H 1990 Gauge Field in Condensed Matter vol I (Singapore: World Scientific) See also Janke W and Kleinert H 1986 Nucl. Phys. B 270399

[27] Ambegaokar V, Halperin B I, Nelson D R and Siggia E D 1980 Phys. Rev. B 211806 Schneider T and Schmidt A 1992 J. Phys. Soc. Japan 612169

[28] Lee D H, Joannopoulos J D, Negele J W and Landau D P 1986 Phys. Rev. B 33450

[29] Isakov S V and Moessner R 2003 Phys. Rev. B 68104409

[30] Zinn-Justin J 1990 Quantum Field Theory and Critical Phenomena (New York: Oxford University Press)

[31] Emig T and Bogner S 2003 Phys. Rev. Lett. 90185701

[32] Binder K and Young A P 1986 Rev. Mod. Phys. 58801

[33] Wessel S and Troyer M 2005 Phys. Rev. Lett. 95127205

Heidarian D and Damler K 2005 Phys. Rev. Lett. 95127206

Melko R G et al 2005 Phys. Rev. Lett. 95127207

Boninsegni M and Prokof'ev N 2005 Phys. Rev. Lett. 95237204 